\title{
CXCR1/2 Inhibitor SX-682
}

National Cancer Institute

\section{Source}

National Cancer Institute. CXCR1/2 Inhibitor SX-682. NCI Thesaurus. Code C150389.

An orally bioavailable, selective and reversible antagonist of C-X-C motif chemokine receptors 1 (CXCR1) and 2 (CXCR2), with potential anti-inflammatory and antineoplastic activities. Upon administration CXCR1/2 inhibitor SX-682 selectively and allosterically binds to CXCR 1 and 2 and inhibits their activation by tumor-secreted chemokines. This inhibits CXCR1/2-mediated signaling, reduces both recruitment and migration of immunosuppressive myeloid-derived suppressor cells (MDSCs) and neutrophils in the tumor microenvironment (TME), inhibits inflammatory processes and abrogates the immunosuppressive-induced nature of the TME. This allows effector cells, such as natural killer (NK) cells and cytotoxic T-lymphocytes (CT Ls), to kill and eliminate cancer cells. This inhibits tumor cell migration, metastasis, angiogenesis and tumor cell proliferation. CXCR1 and 2, G protein-coupled receptor proteins located on myeloid cells and certain tumor cells, play key roles in the immunosuppressive nature of the TME, tumor metastasis, therapy-resistance and myeloid cell suppression. They play a key role in inflammation and their expression is elevated in several inflammatory-driven diseases. 